\title{
Evolution de l'industrie biotech et medtech suisse et influence de l'industrie pharmaceutique sur le système d'innovation
}

The Evolution of the Swiss Biotech and Medtech Industry and the Influence of the Pharma-Shaped Innovation System.

Die Entwicklung der schweizerischen Biotech und Medtech Industrie und Einfluss der Pharmaindustrie auf das Innovationssystem.

\section{Christof Klöpper et Tina Haisch}

\section{OpenEdition}

Journals

Édition électronique

URL : http://journals.openedition.org/rge/1694

DOI : $10.4000 /$ rge. 1694

ISSN : 2108-6478

Éditeur

Association des géographes de l'Est

Édition imprimée

Date de publication : 1 septembre 2008

ISSN : 0035-3213

\section{Référence électronique}

Christof Klöpper et Tina Haisch, « Evolution de l'industrie biotech et medtech suisse et influence de l'industrie pharmaceutique sur le système d'innovation », Revue Géographique de l'Est [En ligne], vol. 48 / 3-4 | 2008, mis en ligne le 02 mars 2010, consulté le 08 septembre 2020. URL : http:// journals.openedition.org/rge/1694; DOI : https://doi.org/10.4000/rge.1694

Ce document a été généré automatiquement le 8 septembre 2020

Tous droits réservés 


\title{
Evolution de l'industrie biotech et medtech suisse et influence de l'industrie pharmaceutique sur le système d'innovation
}

\author{
The Evolution of the Swiss Biotech and Medtech Industry and the Influence of \\ the Pharma-Shaped Innovation System. \\ Die Entwicklung der schweizerischen Biotech und Medtech Industrie und \\ Einfluss der Pharmaindustrie auf das Innovationssystem.
}

Christof Klöpper et Tina Haisch

\section{Introduction}

L'économie suisse est hautement mondialisée et innovante dans plusieurs secteurs; outre le domaine des services financiers, la Suisse s'est fortement spécialisée dans le domaine pharmaceutique et des diagnostics. Dans ce contexte, l'objectif de cet article est d'analyser si, et comment, cette industrie pharmaceutique dominante influence le développement d'industries connexes à l'échelle de la Suisse et de ses régions. L'article suggère que l'industrie pharmaceutique a façonné le développement et la distribution spatiale des secteurs économiques tels que les biotechnologies (biotech) et les technologies médicales (medtech), et que cette influence diffère de manière significative en termes d'extension spatiale. Cette influence est tout d'abord manifeste à l'échelon national, dans la mesure où les technologies médicales et les biotechnologies ont bénéficié d'institutions nationales qui ont été façonnées par l'industrie pharmaceutique. Il est également manifeste que les effets de cette industrie se font sentir par le biais des créations d'entreprises (spin-offs) et des liens d'affaires avec l'industrie pharmaceutique, qui ont conduit à un développement spécifique marqué par une importante concentration de l'industrie pharmaceutique dans la 
région de Bâle. Ces aspects sont évoqués dans l'article en discutant tout d'abord le concept des systèmes d'innovation, puis en détaillant certains aspects de l'économie suisse, avant d'analyser l'industrie biotech et medtech et ses interactions au sein de la Suisse. L'analyse des interactions est basée sur une analyse scientométrique des entreprises biotech et medtech.

\section{Fondements théoriques}

2 Le processus de création de la connaissance est porté par deux idées principales. La première idée s'appuie sur l'importance des interactions entre différents acteurs impliqués dans la production de la connaissance (Dosi 1988 ; Malecki 1990). Cela signifie que les entreprises créent de la connaissance au travers des interactions avec leurs fournisseurs et clients, ainsi qu'avec les universités, les instituts de recherche publics et même avec les concurrents. La seconde idée repose sur le constat que le processus de création de la connaissance dépend du type de connaissance qui a été créé par les entreprises et des infrastructures mises en place pour faciliter ce processus. Par conséquent, la création de la connaissance dépend en premier lieu des trajectoires technologiques et de l'évolution du contexte dans lequel évoluent les entreprises (Nelson et Winter 1974 ; Dosi et Nelson 1994 ; Boschma et Lambooy 1999).

3 Ces deux idées sont au centre du concept de système d'innovation (Freeman 1987; Lundvall 1992; Nelson 1993). Dans cette approche, le cadre institutionnel façonne le processus conduisant à la création de la connaissance. Ce cadre prend un caractère plus ou moins systématique, ce qui signifie que différents acteurs sont fonctionnellement intégrés dans un réseau relativement fermé ou un groupe partageant des institutions identiques (Bathelt et Depner 2003). En outre, ce cadre institutionnel (ou système d'innovation) n'est pas spécifique à un secteur mais susceptible d'influencer plusieurs secteurs économiques.

4 Les systèmes d'innovation diffèrent selon l'échelle géographique, qu'il s'agisse de systèmes d'innovation nationaux (NIS) (Freeman 1987 ; Lundvall 1992 ; Nelson 1993), ou de systèmes d'innovation régionaux (RIS) (Cooke 1992, 1998; Asheim et Isaksen 1997; Asheim et Gertler 2005). En outre, l'impact d'un système d'innovation sur le développement technologique et économique peut également varier; ce qui signifie que ce système peut encourager la croissance d'une industrie particulière ou limiter la croissance ou le progrès technologique d'autres industries.

5 Le débat contemporain sur les systèmes d'innovation s'articule autour de la question de savoir à quelle échelle et selon quelle dimension (technologique ou sectorielle) se manifestent les systèmes d'innovation. Outre l'échelle spatiale, ces systèmes ont été jusqu'ici étudiés du point de vue de la technologie concernée ou du secteur économique impliqué (Breschi et Malerba 1997 ; Carlson 1995). Alors que le système d'innovation régional (RIS) met l'accent sur l'importance des institutions régionales, sur l'enracinement de la connaissance et sur le transfert de cette connaissance au moyen de contacts interpersonnels, le système d'innovation national (NIS) met en avant les institutions formelles telles que les programmes de recherche, les politiques industrielles ou les spécificités du capitalisme comme il est pratiqué dans chacun des pays. Dans les deux approches, les interactions ne se limitent pas nécessairement au territoire régional ou national; au contraire, ces approches relèvent le rôle des contacts internationaux dans la production de la connaissance. Il est cependant 
possible d'analyser les interactions relatives à la connaissance en se limitant à une échelle régionale ou nationale, par exemple en étudiant les effets des programmes nationaux de recherche. Généralement, les approches RIS et NIS ne sont pas mutuellement exclusives, de sorte que plusieurs systèmes peuvent cohabiter dans un même lieu. Il est également possible d'envisager des lieux qui ne sont intégrés à aucun système d'innovation spatialement définis (Malecki et Oinas 1999).

Cet article se concentre sur deux aspects relatifs aux approches des systèmes d'innovation. Le premier aspect concerne l'influence globale de ces systèmes. En partant de l'observation que le système d'innovation suisse a été façonné par une industrie pharmaceutique dominante sur une longue période de temps, l'objectif de cet article est d'analyser comment ce système influence, en retour, le développement d'autres secteurs technologiques et économiques. Dans cet article, les biotechnologies et les technologies médicales sont analysées plus particulièrement pour rendre compte de cette influence. L'intérêt de cette approche est d'étudier comment cette influence s'exprime spatialement. Comme le montrent les chapitres suivants, l'industrie pharmaceutique est fortement concentrée dans une région particulière, alors que son influence s'exprime à l'échelon national tout entier. L'article suggère que celle-ci s'exprime à une échelle régionale et nationale, bien que de fortes différences puissent être observées en ce qui concerne le développement spécifique des biotechnologies et des technologies médicales (chapitre 6), de même que différentes formes d'interactions peuvent être observées entre les acteurs de ces industries (chapitre 7). Ces observations pourraient conduire à la formation d'un RIS dans la région de Bâle, dans le cas où les interactions se limitant à cette région sont plus fortes que celles qui concernent le niveau national ou dans le cas où le processus de création de la connaissance des entreprises de Bâle diffère significativement de celui du reste de la Suisse, par exemple en raison d'institutions spécifiques.

7 L'évolution et les interactions dans les biotechnologies et les technologies médicales sont des cas d'étude particulièrement intéressants dans la mesure où ces deux secteurs sont technologiquement et économiquement reliés à l'industrie pharmaceutique, qu'ils se développent particulièrement bien actuellement et qu'ils présentent des différences entre eux. En effet, alors que les biotechnologies sont une industrie relativement récente et basée sur une connaissance hautement analytique et scientifique, les technologies médicales forment un hybride basé sur une ingénierie qui constitue un savoir-faire reconnu depuis longtemps en Suisse. Etant donné que les données concernant les interactions des entreprises medtech n'ont été récoltées que dans la région de Bâle, cette dimension ne peut être analysée que pour ces entreprises, alors que les interactions qui concernent les entreprises du secteur des biotechnologies seront analysées sur l'ensemble de la Suisse.

\section{Méthodologie}

8 Les données relatives aux entreprises ont été collectées par l'intermédiaire des publications des associations de l'industrie considérée (Unitectra 1999), des agences de promotion économique régionales (Biovalley Basel, Zurich MedNet, Bioalps, Biopolo Ticino) et de la base de données Swiss Life Sciences (Venture Valuation 2006), cette dernière source étant particulièrement adaptée à l'identification des acteurs en question. 
9 Les interactions entre les entreprises sont mesurées par l'intermédiaire des publications effectuées par les entreprises biotech et medtech avec d'autres acteurs (Klöpper 2008). Ces informations ont été collectées grâce à la base de données Web of Science, actualisée pour la dernière fois en janvier 2006. Seules les publications publiées après 1995 ont été considérées. Les interactions ont été mesurées en relevant les localisations géographiques et les affiliations institutionnelles des coauteurs.

10 La méthodologie scientométrique permettant de mesurer les interactions présente plusieurs avantages par rapport à d'autres approches comme les questionnaires ou les entretiens. Tout d'abord, le succès de l'enquête ne dépend pas du taux de réponse ; ensuite, l'ensemble du réseau peut être représenté et non pas seulement une portion du réseau personnel d'une personne enquêtée ; enfin, la reproductibilité de l'enquête est assurée. En outre, l'analyse scientométrique est bien adaptée aux entreprises concernées dans la mesure où l'industrie biotech et medtech publie abondamment. Cela est en partie lié au processus d'approbation des médicaments qui nécessite que les études fassent l'objet de publications, et également au fait que les résultats scientifiques sont évalués par le biais des publications à comités de lecture.

11 Cette méthode présente néanmoins des inconvénients, notamment le fait qu'il n'est pas possible de mesurer la contribution respective de chaque coauteur et que la signification des collaborations scientifiques peut être exagérée, du fait que les publications reçoivent une importance plus grande dans le milieu scientifique en comparaison avec le monde des affaires. En outre, les interactions informelles ne peuvent être mesurées par cette méthode.

\section{L'industrie pharmaceutique et le Système d'innovation suisse}

12 La taille réduite du territoire suisse (16 fois plus petit que celui de la France) pourrait laisser penser que les différences régionales sont peu importantes dans le cas helvétique. Après tout, le pays tout entier est de taille similaire à bien des régions dans d'autres pays. Cependant, les différences institutionnelles sont considérables en Suisse, une conséquence du système politique fédéral qui accorde aux 27 cantons des compétences étendues relatives aux impôts et à la formation par exemple. En outre, la Suisse comporte trois régions linguistiques (allemande, française et italienne) qui présentent des différences culturelles importantes. Par conséquent, même si la plupart des agglomérations suisses sont situées dans un rayon de 100 à 300 kilomètres, la variété institutionnelle est importante.

13 L'industrie pharmaceutique est l'un des piliers de l'économie suisse. Son importance s'est accrue depuis le XVIIIème siècle, dès lors que cette industrie, qui n'était pas encore fondamentalement différente de l'industrie chimique, évolua à partir de la production de colorants chimiques destinés à l'industrie florissante du textile du nord ouest de la Suisse (Bürgin 1958; Bergier 1990). De récentes études conduites par des acteurs privés ou par l'industrie pharmaceutique suisse elle-même montrent que celleci est toujours responsable de plus de la moitié des exportations nationales et présente la croissance de création de valeur la plus élevée de tous les secteurs (Hotz-Hart et al. 2003 ; Elias 2000 ; Credit Suisse 2005 ; Plaut Economics 2005). 
14 L'industrie pharmaceutique a eu une influence considérable sur l'industrie suisse, notamment en façonnant les politiques relatives à la recherche par rapport à ses propres besoins, ce que montre l'orientation très nette des programmes de promotion de la recherche sur la biologie, la chimie et, plus récemment, les bio et nanotechnologies. Plusieurs programmes nationaux de recherche (PP - Programmes prioritaires) aux ressources financières considérables ont été lancés dans le domaine des sciences de la vie (FNS 2002). En conséquence, les universités et les institutions de recherche publiques suisses sont particulièrement actives dans ce domaine (Bergier 1974, 1990; Busset 1997). Cela a également des répercussions importantes pour l'économie nationale dans la mesure où, en Suisse et contrairement à certains autres pays européens, les liens entre les entreprises et les universités sont intensivement développés (Arvantitis et al. 2004).

Une autre influence peut être observée dans le domaine des politiques de la santé. Les prestations liées à la santé sont de très haut niveau mais également très onéreuse en Suisse (OFS 2005; The Economist 2006). En outre, étant donné que les coûts liés à la santé sont couverts par des assurances semi-privées, le marché suisse est demandeur de produits particulièrement haut de gamme. Par suite du lobbying de l'industrie pharmaceutique, la part des médicaments génériques, qui n'intéressent pas particulièrement les entreprises suisses, est nettement plus basse que dans d'autres pays européens. A cela s'ajoute le fait que le prix des médicaments non génériques est plus élevé que dans les pays voisins, que l'usage des médicaments génériques n'est pas encouragé et que les importations de médicaments sont strictement contrôlées.

L'industrie pharmaceutique suisse est fortement concentrée dans le nord-ouest de la Suisse et dans la région de Bâle en particulier (cantons de Bâle-Ville et de Soleure) (OFS 2004 ; Credit Suisse 2004; BAK 2005) (Figure 1). Le seul canton suisse possédant un quotient de localisation plus élevé que 1 , qui ne soit pas localisé dans cette région, est le canton rural de Schaffhouse situé au nord-est de la Suisse, où est installée une filiale de l'entreprise nord-américaine Johnson \& Johnson. La concentration bâloise s'explique par la présence de deux grandes entreprises multinationales actives dans le domaine pharmaceutique, Roche et Novartis, ainsi que par d'autres entreprises présentes dans des secteurs connexes, telles que Syngenta, active dans l'agrobio, les producteurs de produits chimiques Clariant et Ciba, le fabricant Lonza, l'entreprise allemande Bayer qui fabrique ici des médicaments vendus sans ordonnance, de même qu'un grand nombre d'entreprises de services et d'organisation de recherche. Les centres de recherche tels que le Friedrisch-Miescher Institute (une collaboration entre l'Université de Bâle et Novartis), le Roche Institute for Genomic, le Biozentrum et le Pharmazentrum de l'Université de Bâle font de la région bâloise un cluster pharmaceutique et chimique présentant un très large nombre d'acteurs du monde industriel et du monde scientifique. 
Figure 1 : Quotients de localisation des employés de l'industrie pharmaceutique en Suisse

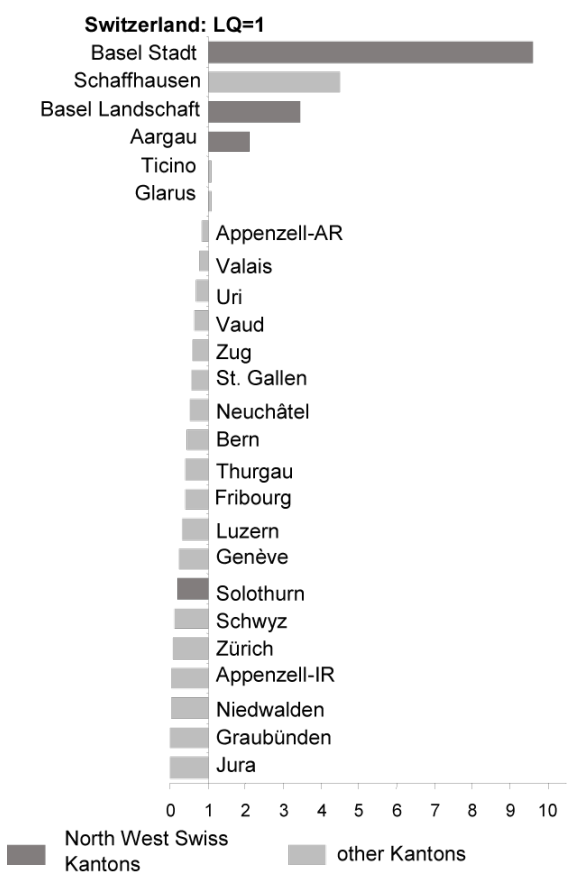

Source : calculs de l'auteur, OFS 2005.

17 A l'échelle nationale, il apparaît que Bâle n'est pas l'unique zone de concentration de l'excellence relative à la recherche. D'autres groupes de recherche actifs dans les sciences de la vie sont également concentrés à Zurich et à Lausanne, où se situent des universités et des instituts financés par la Confédération qui possèdent des centres de recherche dédiés aux sciences de la vie. Ces institutions fédérales sont l'Ecole polytechnique fédérale de Zurich (EPFZ) et l'Institut Paul Scherrer à Zurich ainsi que l'Ecole polytechnique fédérale de Lausanne (EPFL) et l'Institut suisse de recherche expérimentale sur le cancer (ISREC) à Lausanne.

\section{Biotechnologies et technologies médicales : définitions et caractéristiques}

\section{A. Caractéristiques de l'industrie des biotechnologies}

Les biotechnologies sont une industrie récente qui s'occupe du traitement des substances soumises à l'action des agents biologiques (OECD 1999). Il s'agit de technologies travaillant en étroite relation avec d'autres technologies et secteurs, telles que la biologie, la chimie ou les sciences médicales, qui conduisent notamment à des applications relatives aux industries pharmaceutiques, aux producteurs de vaccins et de diagnostics (red biotechnology), aux applications relatives aux agro-sciences (green biotechnology), aux sciences des matériaux et aux sciences environnementales (grey biotechnology). Par suite des relations entre ces différentes technologies, il n'existe pas de définition stricte des biotechnologies et des entreprises qui les utilisent. 
19 Dans ce qui suit, seules les biotechnologies travaillant avec les industries pharmaceutiques, les producteurs de vaccins et de diagnostics (red biotechnology) sont concernées, dans la mesure où les entreprises suisses sont presque exclusivement actives dans ce domaine et parce que ces technologies diffèrent significativement des autres biotechnologies (green et grey) en termes de connaissances déployées et de processus d'innovation. Les biotechnologies dites red sont supposées être plus analytiques et plus volontiers fondées sur les connaissances scientifiques que les autres biotechnologies, qui reposent davantage sur l'ingénierie (Coenen et al. 2005). Une analyse qui mélangerait ces trois types de biotechnologies brouillerait leurs différences relatives aux processus de production de la connaissance.

20 Les biotechnologies sont manifestement l'industrie qui parait la plus proche et qui entretient les relations les plus étroites avec le domaine scientifique (Cooke 2002; Prevezer 2000). Un grand nombre d'entreprises sont directement issues des universités ou s'appuient sur une connaissance produites par les projets de recherche universitaires. Par conséquent, l'investissement public dans la recherche fondamentale des universités et des instituts de recherche est vital pour le développement de l'industrie biotechnologique. Cela explique que cette industrie se développe dans un grand nombre de cas autour des plus grandes universités et des instituts de recherche de pointe (Prevezer 2003; Feldmann et Francis 2003). Une autre propriété des biotechnologies est qu'elles ne créent par de nouveaux marchés, contrairement aux technologies de l'information par exemple, mais qu'elles offrent de nouvelles solutions technologiques à des marchés ou à des secteurs d'activités existants. Dans ces secteurs se trouvent des acteurs bien établis tels que les entreprises pharmaceutiques et certaines institutions telles que les instances approuvant les nouveaux médicaments. Par conséquent, le modèle de développement courant d'une entreprise biotech est de se concentrer sur la recherche et le développement de nouveaux médicaments ou sur de nouvelles technologies et de coopérer avec les entreprises pharmaceutiques dès que des tests cliniques doivent être entrepris ou si un produit doit être commercialisé. Les formes de coopération les plus typiques concernent les licences relatives aux droits de propriété ou la vente de l'entreprise à une entreprise pharmaceutique. De ce fait, les interactions entre les entreprises biotechnologiques et pharmaceutiques sont courantes (voir Zeller 2004 en ce qui concerne la coopération entre entreprises pharmaceutiques suisses et américaines).

21 La connaissance créée et appliquée dans l'industrie biotechnologique tend à être très spécifique, ce qui signifie qu'une technologie particulière peut parfois être maîtrisée par quelques scientifiques ou par quelques entreprises uniquement. Cela s'explique par le fait que les applications industrielles se trouvent dans leurs premiers stades de développement et demeurent proche de la recherche. En outre, les petites entreprises de biotechnologies sont généralement très spécialisées sur un type ou sur une famille de médicaments, ou encore sur une technologie particulière. Par conséquent, cette connaissance très spécialisée ne peut être générée par n'importe quelle entreprise ou scientifique, ce qui implique que les interactions entre les acteurs de cette industrie peuvent significativement différer en termes d'objectifs et d'affiliation. Dans les premiers stades de la recherche et développement (R\&D) des médicaments, les entreprises biotech interagissent principalement avec les universités et les institutions publiques de recherche, avec d'autres entreprises semblables à elles et avec des 
entreprises pharmaceutiques. Cet ensemble est usuellement appelé la « sainte trinité » dans l'innovation biotechnologique (Cooke 2005).

De très nombreux travaux ont traité de la manière dont l'industrie biotechnologique se développe spatialement depuis une dizaine d'années. Ces travaux ont montré que les biotechnologies et leurs applications économiques tendent à adopter la forme d'un cluster (voir par exemple Prevezer 1997; Zeller et Ossenbrügge 2002; Cooke 2003; Lawton Smith 2005). Ces clusters sont cependant censés différer des clusters orientés par le marché, du fait qu'ils présentent un nombre d'interactions input-output plus faible à l'intérieur du cluster (Cooke 2005). Cela s'explique par le fait que la connaissance relative aux biotechnologies est très spécifique et ne peut être présente dans l'ensemble des clusters, et par le fait qu'un grand nombre d'entreprises ne possèdent pas encore de produit présent sur le marché. Par conséquent, les relations input-output qui se tissent entre les entreprises actives dans la production ne peuvent être un avantage crucial de localisation dans un cluster. En outre, la compétition basée sur les produits ne peut constituer le moteur principal du développement comme cela est suggéré dans les clusters de Porter (1990), bien qu'une compétition potentielle pour d'autres facteurs tels que le capital-risque ou le capital humain puisse exister (Niosi et Bas 2003 ; Cooke 2005). En revanche, les raisons qui poussent les entreprises à se regrouper en clusters sont liées à la présence d'instituts de recherche, d'universités et d'institutions annexes qui facilitent le transfert de connaissance du domaine scientifique au monde des affaires (Cortright et Mayer 2002). Cooke (2002) suggère que la connaissance et les entreprises liées aux biotechnologies sont concentrées dans un nombre limité de lieux dans le monde, appelés des méga centres. Les mégacentres les plus importants seraient notamment Boston ou la région de San Francisco (Bay Area) aux Etats-Unis, ainsi que Cambridge et Munich en Europe.

\section{B. Caractéristiques de l'industrie medtech}

Différents termes tels que «technologies médicales», "medtech» ou «services médicaux » sont utilisés pour décrire l'industrie et les sciences qui s'occupent des applications médicales. Cet article considère les technologies médicales, dites medtech, comme "...the part of life sciences that covers the research, development, production and marketing of systems and devices for medical applications for humans and animals » (Venture Valuation 2006). Ces technologies produisent par exemple des pompes à insuline, des tissus et des os destinés aux transplantations, des cartilages ou des implants dentaires.

Le fait que la définition des technologies médicales soit large et que le nombre de soussecteurs soit important reflète l'hétérogénéité de la connaissance et des technologies employées dans cette industrie. Il s'agit en particulier de connaissances issues de plusieurs champs scientifiques, tels que la physique, la chimie, la médecine et l'ingénierie. Des compétences développées dans le cadre de l'industrie électronique, des machines à haute précision, des technologies des matériaux et des surfaces ainsi que dans l'ingénierie mécanique sont mobilisées pour développer les produits de l'industrie des technologies médicales. Récemment, les technologies médicales, pharmaceutiques et biotechnologiques sont devenues de plus en plus complémentaires et il est attendu que les produits issus de l'industrie medtech soient utilisés pour traiter directement les cellules cancéreuses à l'aide d'agents biologiques (Clayton-Matthews 2001). 

spécialisation de l'économie suisse en faveur de l'industrie pharmaceutique a influencé le développement des industries medtech et biotech. A cela s'ajoute le fait que cette influence diffère selon les échelles géographiques considérées, ce qui signifie que les entreprises de la région de Bâle se sont développées différemment de leurs consœurs du reste de la Suisse. Cette hypothèse est analysée d'abord pour l'industrie biotech et ensuite pour l'industrie medtech.

\section{A. Evolution de l'industrie biotech en Suisse}

Analyser l'évolution de l'industrie biotech suisse ne nécessite qu'un bref historique. En effet, cette industrie ne s'est développée qu'à partir du milieu des années 1990 en Suisse, soit environ dix ans plus tard qu'aux Etats-Unis ou qu'en Grande-Bretagne. Depuis son origine, cependant, le développement de cette industrie a été rapide, à tel point qu'Ernst \& Young (2003a et 2003b) parle de la Suisse comme de l'épicentre

Revue Géographique de l'Est, vol. 48 / 3-4 | 2008 
(hotspot) des biotechnologies en Europe. Plusieurs entreprises issues des universités s'y sont établies, tandis que les entreprises pharmaceutiques et de services se spécialisaient dans les produits ou dans les services liées aux biotechnologies. La part élevée d'entreprises qui se consacrent à la recherche exploratoire (en opposition à celles qui se destinent à la recherche technologique) et le grand nombre de médicaments testés sont considérés comme des atouts de l'industrie biotechnologique suisse. Aujourd'hui, $46,8 \%$ des entreprises de biotechnologie sont spécialisées dans la découverte de nouveaux médicaments, la bioinformatique ou les biopuces, alors que $53,2 \%$ des entreprises offrent des services, de la recherche contractualisée ou des technologies liées aux médicaments. En raison des nombreuses définitions qui entourent les termes « biotechnologie » et " entreprises de biotechnologie », le nombre d'entreprises concernées varie selon les sources fournies par Ernst \& Young (2003a), Unitectra (1999) ou Ventura Valuation (2006).

Les entreprises concernées ne sont pas localisées uniquement dans la région de Bâle et se trouvent également à Zurich, Lausanne, Genève et, dans une moindre mesure, à Berne (Figure 2). En conséquence, la structure spatiale de cette industrie varie de celle qui a été décrite pour l'industrie pharmaceutique et dont on se souvient qu'elle était fortement concentrée dans la région bâloise. Les localisations choisies par les entreprises biotech varient grandement selon que sont considérés la structure ou le contexte industriel dans lequel évoluent les établissements concernés (Kollreuter 2005).

Figure 2. Localisation des entreprises biotech en Suisse

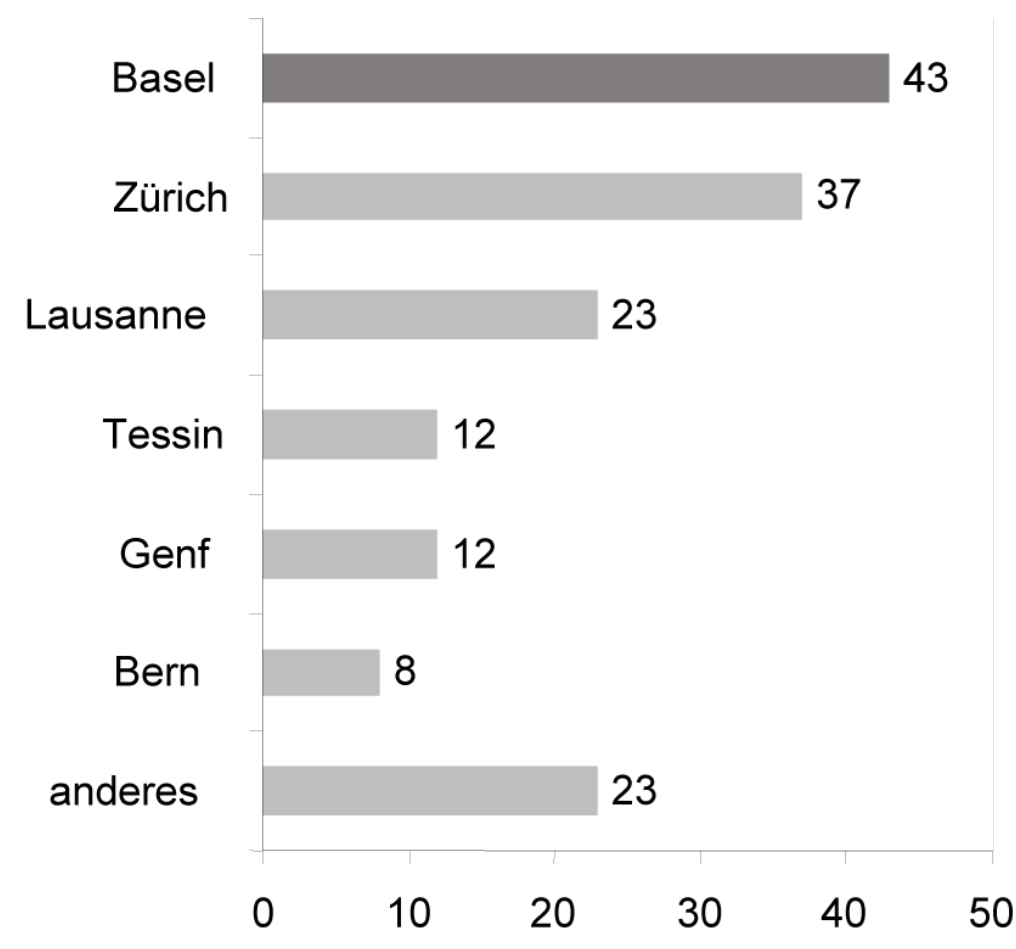

Source : de l'auteur.

Il est frappant de constater que la région de Bâle compte peu d'entreprises issues du monde universitaire en comparaison avec les autres villes suisses. Seules 9 des 19 entreprises sont directement issues des universités à Bâle, tandis que les autres ont été 
créées durant la fin des années 1990 à partir d'autres entreprises du secteur biotechnologique, en particulier Novartis et Roche. Cela peut être expliqué par le contexte industriel de cette période au cours de laquelle les deux multinationales pharmaceutiques précédemment mentionnées furent soumises à de profondes restructurations et furent conduites à réévaluer, abandonner ou externaliser certains projets de développement de médicaments. Ces restructurations étaient liées à la prise de contrôle de l'entreprise pharmaceutique allemande Boehringer-Mannheim par Roche en 1997, et à la création de Novartis par la fusion de Sandoz et Ciba-Geigy, deux entreprises pharmaceutiques bâloises en 1996 (Zeller 2001).

Par suite de ce contexte industriel, les entreprises biotech bâloises différent de leurs consœurs du reste du pays, dans la mesure où elles sont plus grandes et possèdent des produits plus proches ou déjà placés sur le marché. Un autre facteur expliquant la faible proportion d'entreprises issues de l'université peut être lié au fait que les universités et les instituts de recherche bâlois ne sont autant spécialisés sur les biotechnologies que ceux des écoles polytechniques fédérales de Lausanne et de Zurich. En effet, durant les années 1990, l'université de Bâle a plutôt été au service de l'industrie pharmaceutique, en se focalisant sur les technologies traditionnelles de la chimie et de la pharmacie. Cela peut naturellement être interprété comme le résultat de l'influence des plus grandes entreprises bâloises, que sont Novartis et Roche, sur l'universités et les instituts de recherche publics à Bâle et leur intérêt manifeste pour la recherche appliquée et immédiate, typique des années 1990 (Hicklin 2005). Cet intérêt s'est concrétisé par la création du Friedrich-Miescher Institute, une importante infrastructure de recherche dans le domaine de la recherche sur les médicaments financée et contrôlée conjointement par Novartis et le canton de Bâle, ou par la création d'un certain nombre d'instituts universitaires financés par Novartis ou Roche (Uni News 2006).

Contrairement à la région de Bâle, Zurich et Lausanne n'accueillent aucune entreprise issue du monde industriel, mais plutôt de jeunes entreprises issues des universités et des écoles polytechniques fédérales. En outre, 10 des 13 entreprises nouvellement créées par le Programme prioritaire Biotechnologie (Biotech) financé par la Confédération sont localisées à Zurich ou à Lausanne, alors qu'une seule entreprise est située à Bâle. Tandis que Lausanne est une agglomération de taille relativement réduite, Zurich est la plus grande ville de Suisse et possède une économie spécialisée dans les services financiers et, dans une moindre mesure, dans les industries de haute technologie, telles que les technologies de l'information, ou les medtech. Contrairement à Lausanne et à Zurich où les entreprises sont plutôt de petite taille et où aucun très grand acteur biotechnologique n'est présent, Berne et Genève sont dominées par de grandes entreprises: BernaBiotech domine la scène dans la capitale tandis que Serono, qui est la troisième plus grande entreprise biotech du monde, a une position dominante dans la Cité de Calvin. Au Tessin, finalement, les entreprises concernées ne fournissent que des services.

L'analyse de l'évolution de l'industrie des biotechnologies en Suisse permet de mettre en évidence deux influences exercées par l'industrie pharmaceutique. En premier lieu, cette industrie produit un effet accélérateur sur le système d'innovation suisse et particulièrement sur la recherche publique, depuis plusieurs décennies. Cette influence se manifeste par le grand nombre d'entreprises qui sont issues des projets spécialisés conduits par les universités et le Fonds national suisse de la recherche scientifique 
(FNS). Elle affecte l'ensemble de la Suisse et particulièrement les régions dans lesquelles se trouvent les écoles polytechniques et les instituts de recherches financés par la Confédération, à Zurich et à Lausanne. En second lieu, l'industrie pharmaceutique joue le rôle d'incubateur pour les entreprises de biotechnologie. Cet effet est plus direct et n'affecte que la région de Bâle. En outre, cet effet ne s'est fait sentir, pour les entreprises analysées, que pendant une période de temps limitée à cinq ans au cours de laquelle des changements majeurs ont eut lieu dans l'industrie pharmaceutique suisse. En raison de ces deux influences, les entreprises bâloises se sont développées d'une manière différente par rapport aux autres entreprises en Suisse.

\section{B. Evolution de l'industrie medtech en Suisse}

L'industrie medtech est particulièrement importante pour l'économie suisse, dans la mesure où elle génère un chiffre d'affaires de 5 milliards de US\$ et emplois 40000 personnes en 2005 (soit 2\% des employés du pays). Plusieurs grands acteurs tels que Roche Diagnostics, Stramann, Phonak, Synthes et Nobel BioCare ont été fondés et/ou possèdent un siège social ou une importante activité en Suisse.

Contrairement à l'industrie pharmaceutique, mais comme les biotechnologies, l'industrie medtech n'est pas uniquement concentrée à Bâle. De fortes densités d'entreprises du domaine medtech peuvent être observées à Zurich, à Berne ou dans la région de Genève-Lausanne (Figure 3).

Figure 3. Localisation des entreprises medtech en Suisse

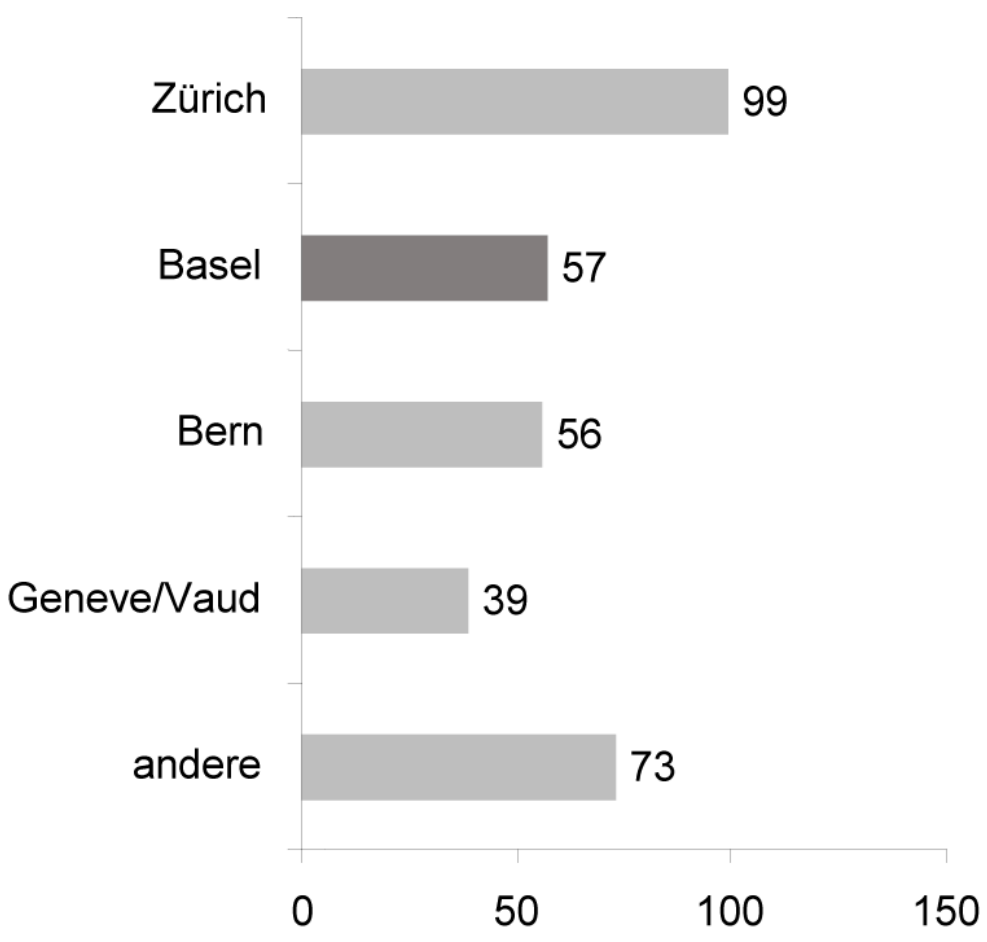

Source : de l'auteur; Venture Valuation 2006

Bâle et le Jura sont considérés cependant comme les localisations les plus importantes de cette industrie en raison du fait que ces régions accueillent de grandes entreprises et 
possèdent une longue tradition dans le domaine. Près d'un tiers de la force de travail des medtech est localisée dans ces régions : Roche Diagnostics (7860 employés en 2005), Synthes (environ 1500), Straumann (750), Ionbond (environ 650), Mathys (300). Les entreprises medtech de Zurich et de la région de Genève-Lausanne sont plus petites et bien souvent récemment créées.

L'évolution de l'industrie medtech en Suisse est influencée par plusieurs facteurs. Il s'agit en premier lieu des développements technologiques qui ont conduit les entreprises de l'industrie des instruments de précision à se diversifier dans les technologies médicales. La plupart des grandes entreprises contemporaines ont été par le passé des sous-traitants de l'industrie horlogère dans le Jura suisse, produisant par exemple différents alliages non-magnétiques et résistants aux chocs (Straumann), des machines (Mathys) ou des pièces pour l'industrie automobile (Weidmann Plastics Technology). L'atout principal de ces entreprises durant leur entrée dans l'industrie medtech fut leur spécialisation dans les produits de haute précision et de haute qualité et dans la réalisation de séries limitées. Depuis les années 1960, ces entreprises ont commencé à collaborer avec des institutions de recherche médicale, en particulier avec celles qui possédaient une spécialisation en chirurgie. Ce processus a conduit à réunir les connaissances médicales et l'ingénierie et a abouti par exemple au développement de produits osthéosynthétiques. Le virage en faveur des technologies médicales fut principalement motivé par les perspectives de croissance du secteur medtech. Le développement d'appareils médicaux fut rendu possible par la présence d'une maind'œuvre qualifiée et expérimentée active dans l'industrie de précision, l'électromécanique et les technologies des matériaux.

39 En second lieu, l'industrie medtech a été influencée par les consommateurs, en raison de la haute qualité et du coût élevé du système de santé suisse. Comme l'a suggéré le chapitre 4 , cette situation résulte de l'influence durable de l'industrie pharmaceutique sur les politiques de santé nationales. Durant les dernières décennies, les entreprises medtech de Suisse ont opéré à partir d'un marché intérieur caractérisé par des prix élevés qui a permis d'investir et d'innover dans ce secteur et qui peut, selon Porter (1990: 89), donner un avantage important à certaines entreprises sur leurs concurrents.

40 En troisième lieu, et dans une moindre mesure, l'industrie medtech a été influencée par la recherche publique. Les fondations dédiées à la science que sont le Fonds national suisse de la recherche scientifique (FNS), destiné à la recherche fondamentale, et la Commission pour la technologie et l'innovation (CTI), orientés vers la recherche appliquée, ont lancé de grands projets relatifs à l'industrie medtech. Cela constitue cependant un phénomène récent (depuis 1997) qui peut être considéré plutôt comme une conséquence que comme une cause du succès de l'industrie medtech, particulièrement dans le cas bâlois.

41 Au final, en dépit de localisations similaires, il est difficile de mettre clairement en évidence l'influence que pourrait avoir l'industrie pharmaceutique sur l'industrie medtech suisse. Il existe probablement une influence via le système de santé mais de plus amples recherches sont nécessaires pour valider cette hypothèse. 


\section{Interactions dans l'industrie biotech et medtech}

42 Ce chapitre analyse les interactions des entreprises biotech et medtech. Ce faisant, il permet de tester la seconde hypothèse de ce travail, selon laquelle les interactions entre les secteurs biotech et medtech sont influencées par l'industrie pharmaceutique. En conséquence, il est attendu que les entreprises bâloises interagissent différemment des autres entreprises du pays, c'est-à-dire que les interactions de ces entreprises ont une dimension plus régionale et que l'industrie pharmaceutique bâloise possède une position centrale dans la structure des interactions pour l'ensemble des entreprises biotech et medtech en Suisse.

\section{A. Interactions des entreprises biotech en Suisse}

Les interactions sont analysées séparément, qu'il s'agisse des principales localisations des entreprises biotech ou des affiliations institutionnelles des coauteurs. Comme mentionné dans le chapitre 3, les coauteurs sont considérés comme des partenaires interagissant dans les entreprises considérées. Les affiliations institutionnelles sont référencées en tant qu'« universités et institutions publiques de recherche » (ci-après universités), "entreprises de biotechnologie » et "entreprises pharmaceutiques et chimiques ». Ces dernières entreprises sont incluses dans l'analyse, compte tenu du fait qu'elles ne peuvent très souvent pas être isolées des entreprises pharmaceutiques. Les hôpitaux et les entreprises de services telles que les medical writers ou les organisations de recherche contractuelle ne sont pas incluses dans l'analyse du fait que les interactions qui concernent ces acteurs sont typiques des phases tardives de développement des produits, qui ne sont pas abordées ici. Deux grandes entreprises bien installées dans le milieu publient un nombre particulièrement important d'articles : Serono à Genève et BernaBiotech à Berne. Dans ces deux villes, peu d'autres entreprises sont actives dans la publication et, de ce fait, il est impossible de mesurer leurs interactions par la méthode scientométrique. Les entreprises du Tessin sont, comme mentionné précédemment, plutôt des firmes actives dans les services liés aux médicaments (elles développent des solutions permettant aux médicaments d'atteindre l'endroit approprié du corps). Ces entreprises ne publient presque pas et sont par conséquent analysées séparément. En ce qui concerne l'analyse des interactions entre entreprises biotech, 1355 publications ont été analysées, parmi lesquelles 1036 possédaient un coauteur situé en dehors de l'entreprise considérée.

Comme l'a suggéré le chapitre 5 , les interactions entre les entreprises biotech et les universités ou les instituts de recherche publics sont particulièrement importantes du fait de la proximité de cette industrie avec la science fondamentale, ce qui se confirme par le fait que $62,5 \%$ des coauteurs sont affiliés à une université. Cependant, cette proportion élevée révèle également un biais de la méthode scientométrique, laquelle surestime le nombre d'universités partenaires en comparaison avec les partenaires industriels (voir chapitre 4).

La structure des affiliations des coauteurs diffère significativement selon les localisations des entreprises considérées : les entreprises bâloises interagissent moins fréquemment avec les partenaires universitaires que les entreprises du reste de la Suisse (50,9\% contre $62,5 \%$ en moyenne nationale), mais les contacts avec les industriels y sont plus fréquents (19,6\% de tous les partenaires contre $7,9 \%$ en moyenne nationale) 
(Figure 4). Ces résultats s'expliquent partiellement par le contexte bâlois marqué par des entreprises issues du monde industriel.

Figure 4. Interactions entre entreprises biotech et universités/instituts publics de recherche

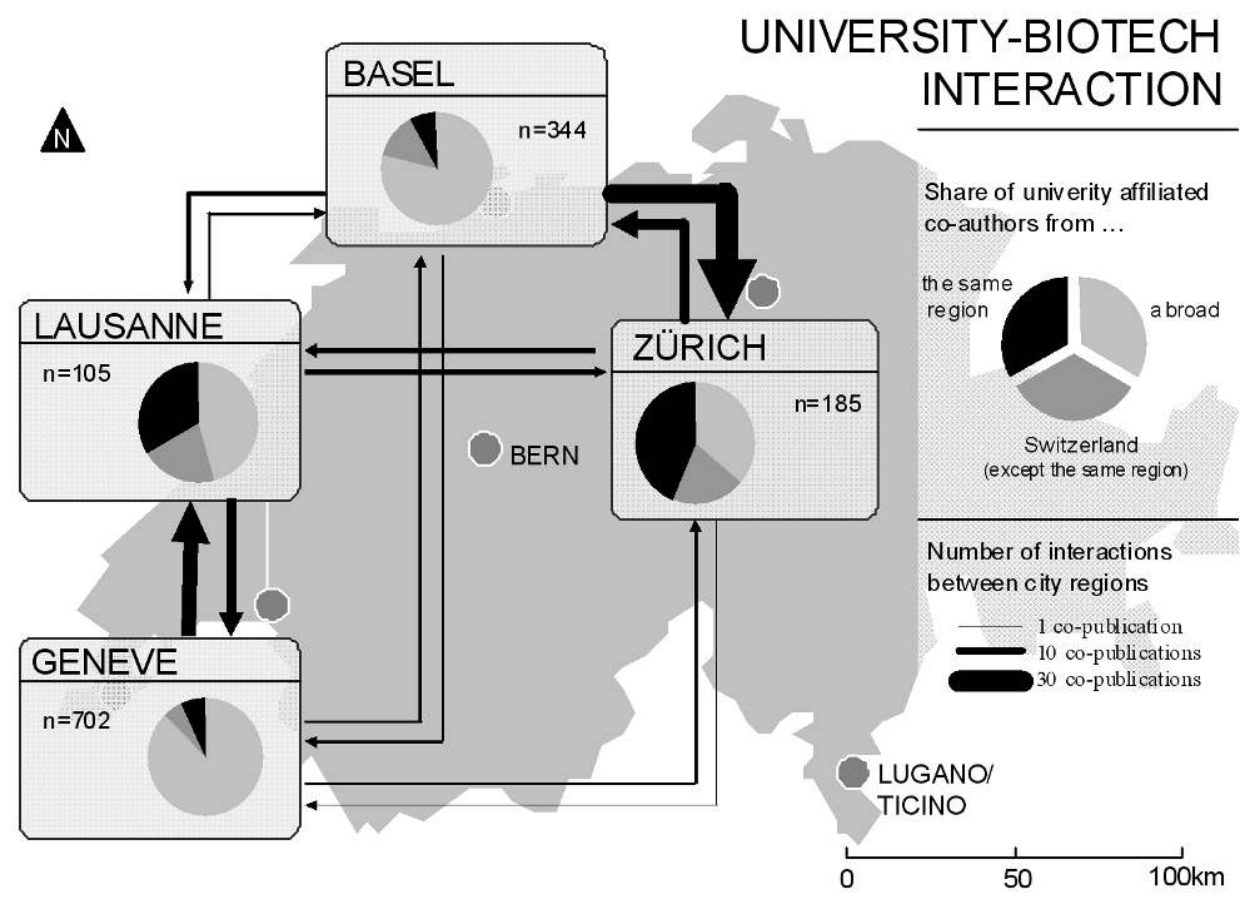

Source : calculs de l'auteur à partir de Web of Science 2006 internationale, qui se marque par le fait que seuls $26,6 \%$ des partenaires universitaires sont localisés en Suisse. La plus grande partie de ces partenaires étrangers sont localisées aux Etats-Unis (23,4\%), en Allemagne (11,8\%), en France (8,9\%) et en GrandeBretagne (6,5\%). Les entreprises bâloises et genevoises présentent la proportion la plus élevée de partenariats noués avec les universités étrangères, alors que les entreprises de Zurich et de Lausanne sont plus enclines à travailler avec des partenaires universitaires suisses, notamment ceux de leur région (voir Figure 4). Cela s'explique en premier lieu par la forte proportion d'entreprises issues des universités, qui maintiennent des relations avec leurs institutions d'origine et en second lieu par la diversité et la qualité des institutions de recherche localisées dans ces villes (voir chapitre 3). En outre, les co-publications montrent que les universités et instituts de recherche bâlois collaborant rarement dans l'échantillon analysé. En ce qui concerne les interactions entre les entreprises biotech et les universités à l'échelle nationale, deux flux d'interactions principaux peuvent être observés : ceux qui lient Zurich et Bâle dans la partie alémanique, et ceux qui lient Lausanne et Genève dans la partie francophone (Tableau 1). Cette particularité pourrait être expliquée par la barrière linguistique, mais étant donné que l'anglais constitue la langue de travail dans un grand nombre d'entreprises à la fois dans la partie alémanique et francophone, il est probable que d'autres variables entrent en ligne de compte, telles que les différents systèmes éducatifs et marchés de l'emploi.

Les interactions entre les entreprises pharmaceutiques et biotech sont bien moins fréquentes que celles entre les universités et les entreprises biotech. Seuls $7,7 \%$ des 
coauteurs travaillent pour les entreprises pharmaceutiques et plus de la moitié $(57,7 \%)$ de ces coauteurs sont localisés en Suisse. Les autres localisations des partenaires industriels sont les Etats-Unis et l'Allemagne. Plus de 90\% des partenaires affiliés aux entreprises pharmaceutiques en Suisse sont basés à Bâle, ce qui résulte de la distribution de ces entreprises sur le territoire national et de la position dominante de Bâle dans ce secteur comme l'a montré la Figure 1.

Tableau 1. Interactions entre les entreprises biotech et les entreprises pharmaceutiques

\begin{tabular}{lcccc}
\multicolumn{5}{c}{$\begin{array}{l}\text { coauthors affiliated with pharmaceutical companies in ... } \\
\text { (in \% of co-authors of companies from location) }\end{array}$} \\
author from ... & $\mathrm{n}$ & abroad & Switzerland & in Basel \\
\hline Basel & 127 & 41.73 & 58.27 & 55.91 \\
Zürich & 6 & 66.67 & $-^{*}$ & $-^{*}$ \\
Genève & 26 & 42.31 & 57.69 & 50.00 \\
Lausanne & 7 & 71.43 & 28.57 & $-^{*}$ \\
Bern & 5 & 60.00 & $-{ }^{*}$ & 68.75 \\
other & 16 & 31.25 & 68.75 & 48.13 \\
total Switzerland & 187 & 43.32 & 56.68 & \\
*not calculated if the number is 2 or lower
\end{tabular}

Source : calculs de l'auteur à partir de Web of Science 2006.

Des différences entre les entreprises basées en Suisse existent du point de vue de la fréquence des liens entre entreprises biotech et pharmaceutiques ainsi qu'en termes de rayonnement spatial des interactions. Les entreprises biotech bâloises comptent pour près de 70\% (127 sur 187) des publications coécrites dans l'industrie pharmaceutique, ce qui est partiellement dû au fait que près de la moitié des entreprises biotech présentes à Bâle sont issues de l'industrie, en particulier de Roche et Novartis, pour lesquelles travaillent un grand nombre de coauteurs de l'industrie. En outre, comme l'a montré le chapitre 6 , les entreprises bâloises tendent à produire des produits qui sont proches du marché; par conséquent, les coopérations avec les entreprises pharmaceutiques pourraient constituer un indicateur de la collaboration mise en œuvre dans les phases tardives du développement des produits.

Outre les entreprises bâloises, seule Serono à Genève présente un nombre important de partenaires dans les entreprises pharmaceutiques (26). Bâle et Genève possèdent plus de partenaires étrangers que les entreprises situées dans les autres villes du pays. De ce fait, les entreprises de ces deux villes sont fortement intégrées dans les relations d'affaires et sont plus internationales que les entreprises de Lausanne et de Zurich, qui coopèrent rarement avec les entreprises pharmaceutiques et, lorsqu'elles le font, tendent à travailler avec un coauteur situé en Suisse plutôt qu'à l'étranger. 
Tableau 2. Interactions entre les entreprises biotech

\begin{tabular}{|c|c|c|c|c|}
\hline \multicolumn{5}{|l|}{ author } \\
\hline from... & $\mathrm{n}$ & abroad & Switzerland & the same agglomeration \\
\hline Basel & 23 & 82.61 & 17.39 & 13.04 \\
\hline Zürich & 10 & 50.00 & 50.00 & 30.00 \\
\hline Genève & 40 & 77.50 & 22.50 & 22.50 \\
\hline Lausanne & 9 & 88.89 & 11.11 & 11.11 \\
\hline Bern & 4 & 100.00 & 0 & 0 \\
\hline other & 10 & 70.00 & 30.00 & $--^{\star \star}$ \\
\hline total Switzerland & 97 & 76.29 & 23.71 & $--^{\star \star}$ \\
\hline
\end{tabular}

Source : calculs de l'auteur à partir de Web of Science 2006. dans cette analyse. Seuls 97 des 2431 coauteurs (4\%) sont affiliés à une entreprise biotech. Cela est partiellement dû à la méthodologie employée, qui mesure seulement les contacts scientifiques formels. Les entreprises biotech pourraient, par exemple, interagir en partageant des infrastructures ou par l'intermédiaire de discussions scientifiques ou d'affaires, ce qui ne pourrait être mesuré ici. Les interactions formelles sont quant à elles sans doute motivées par les technologies possédées par les entreprises, qui peuvent faire l'objet d'échange entre elles. Une forme habituelle de coopération intervient par exemple lorsque des entreprises spécialisées dans la découverte et des entreprises plutôt focalisées sur les technologies décident de travailler ensemble, la seconde contribuant à fournir les connaissances nécessaires au développement d'un médicament particulier. Les entreprises orientées vers les aspects technologiques ont été particulièrement encouragées par les investisseurs à collaborer de cette manière durant la phase de restructuration des biotechnologies, jusqu'à l'éclatement de la bulle high-tech en 2001. Une proportion importante des partenaires des firmes biotech sont basés en dehors de la Suisse (23,7\% des coauteurs), notamment aux Etats-Unis (25,8\%), en Grande-Bretagne (16,7\%) ou en Allemagne (14,6\%). En raison du nombre limité d'observations, une analyse systématique des différences entre les concentrations observées en Suisse n'est pas réalisable. Cependant, il est possible de mettre en évidence le fait que les entreprises localisées à Genève et à Bâle interagissent plus fréquemment avec d'autres entreprises biotech que celles de Lausanne ou de Zurich, et tendent à interagir avec des entreprises étrangères (Tableau 2). En outre, on note que 14 des 19 coauteurs basés en Suisse dans les entreprises biotech sont localisés dans la même région que l'entreprise avec laquelle ils travaillent, ce qui pourrait résulter d'un partage des laboratoires, de contacts informels ou de spin-offs créées à partir d'un même institut, ce qui, en retour, accroît la probabilité que la connaissance soit ensuite partagée de manière formelle. A cela s'ajoute le fait que les scientifiques sont encouragés à fréquenter des collègues proches en raison des spécificités des marchés du travail locaux.

L'analyse des interactions des entreprises biotech basées en Suisse révèle un comportement particulier pour celles qui sont localisées à Bâle. Ces entreprises, à la différence de celles de Lausanne et de Zurich, tendent à avoir plus d'interactions au niveau régional et plus fréquemment avec des partenaires universitaires. Les firmes de Bâle, et dans une moindre mesure de Genève, coopèrent plus fréquemment avec des partenaires industriels et ont une probabilité supérieure de posséder des partenaires étrangers. Ces résultats convergent avec ceux qui ont été présentés dans le chapitre 6, à

Revue Géographique de l'Est, vol. 48 / 3-4 | 2008 
savoir que les entreprises bâloises et genevoises sont plus ancrées dans l'industrie que dans le milieu scientifique parce qu'elles sont plus matures que celles de Zurich et de Lausanne. En outre, l'analyse montre que Bâle constitue le centre des interactions avec les entreprises pharmaceutiques, ce qui est naturellement une conséquence de la position dominante de l'industrie pharmaceutique bâloise en Suisse. Il y a trop peu d'entreprises de ce secteur en dehors de Bâle qui puissent jouer un rôle significatif dans les interactions observées. En revanche, les interactions avec les entreprises biotech et les universités ne sont pas particulièrement fréquentes à Bâle, ce qui laisse penser que la seconde hypothèse est partiellement vérifiée. D'une part, il est vrai que Bâle diffère des autres localisations du pays et que les interactions avec les entreprises pharmaceutiques sont concentrées dans cette ville. D'autre part, il n'est pas vérifié que Bâle constitue réellement un lieu de concentration des interactions entre les entreprises biotech et les universités, et entre les entreprises biotech elles-mêmes. En outre, l'hypothèse selon laquelle les interactions des entreprises bâloises sont plus fortement limitées au cadre régional que celles des autres entreprises du pays, du fait de la «sainte trinité » caractéristique du développement des médicaments dans la région, n'est pas vérifiée.

\section{B. Interactions des entreprises biotech bâloises}

Les interactions qui concernent les entreprises biotech situées à Bâle sont analysées en termes d'affiliation institutionnelle et de localisation des coauteurs concernés. Les affiliations sont les suivantes: "universités, institutions publiques de recherche et hôpitaux " (ci-après universités) et " autres entreprises medtech et pharmaceutiques, autres entreprises et instituts de recherche privés » (ci-après entreprises). Presque tous les hôpitaux analysés (90\%) sont des hôpitaux universitaires ou des centres médicaux publics.

Seules 11 des 57 entreprises medtech de la région de Bâle ont publié durant la période allant de 1981 à 2006. Deux entreprises ont eu une fréquence de publication particulièrement importante et représentent $69,7 \%$ des co-publications de la région de Bâle : Straumann, une firme basée dans le canton de Bâle-Campagne qui compte pour $41,4 \%$ des co-publications, et la Fondation Robert Mathys, un institut de recherche privé localisé depuis 2004 à Bâle, après avoir déménagé du canton de Soleure. Ces deux institutions forment des acteurs bien établis qui maintiennent des relations étroites avec le milieu scientifique: Straumann participe au réseau indépendant ISI, qui regroupe 400 spécialistes du monde entier travaillant sur les technologies médicales, alors que la Fondation Robert Mathys développe ses activités de recherche en partenariat étroit avec Mathys SA, sa maison mère.

Comme l'a montré le chapitre 5.2, les relations des entreprises medtech avec les universités sont particulièrement importantes durant les phases de recherche préalable et de test des produits, durant lesquelles une forme de connaissance plus codifiée est échangée. A ce titre, les partenaires de recherche des entreprises medtech considérées sont localisées à l'échelle mondiale : 196 des 264 partenaires universitaires des entreprises medtech sont localisées à l'étranger, en particulier aux Etats-Unis et en Allemagne.

Les interactions suisses sont focalisées en direction de Zurich (EPFZ et Université de Zurich), Berne (hôpital universitaire local) et de Lausanne-Genève (EPFL). Neuf co- 
publications sur 15 écrites par des auteurs en provenance des " autres régions » sont publiées en collaboration avec l'Association for the Study of Internal Fixation, un organisme sans but lucratif basé à Davos dans les Alpes suisses. En dépit de la qualité des instituts de recherche et des universités bâloises, presque aucune co-publication n'est observée dans le domaine des sciences de la vie dans la région de Bâle. L'une des raisons pourrait être qu'aucune spécialisation dans les technologies médicales n'existe au sein des institutions de recherche de cette région. Une autre interprétation pourrait être que les structures de recherche ne peuvent se développer dans ce domaine en raison de l'influence de l'industrie pharmaceutique sur l'université. Cela ne signifie pas que l'industrie pharmaceutique soit directement impliquée, mais que la tradition historique et l'appui financier de cette industrie auraient contribué à développer un champ de recherche très orienté vers le développement pharmaceutique. A l'heure actuelle, en 2006, un léger changement peut être observé dans le paysage de la recherche, dans la mesure où de nouveaux instituts et de nouveaux diplômes universitaires sont mis en place dans le domaine des sciences appliquées.

Figure 5. Interactions entre les entreprises medtech et les universités/instituts de recherche publics

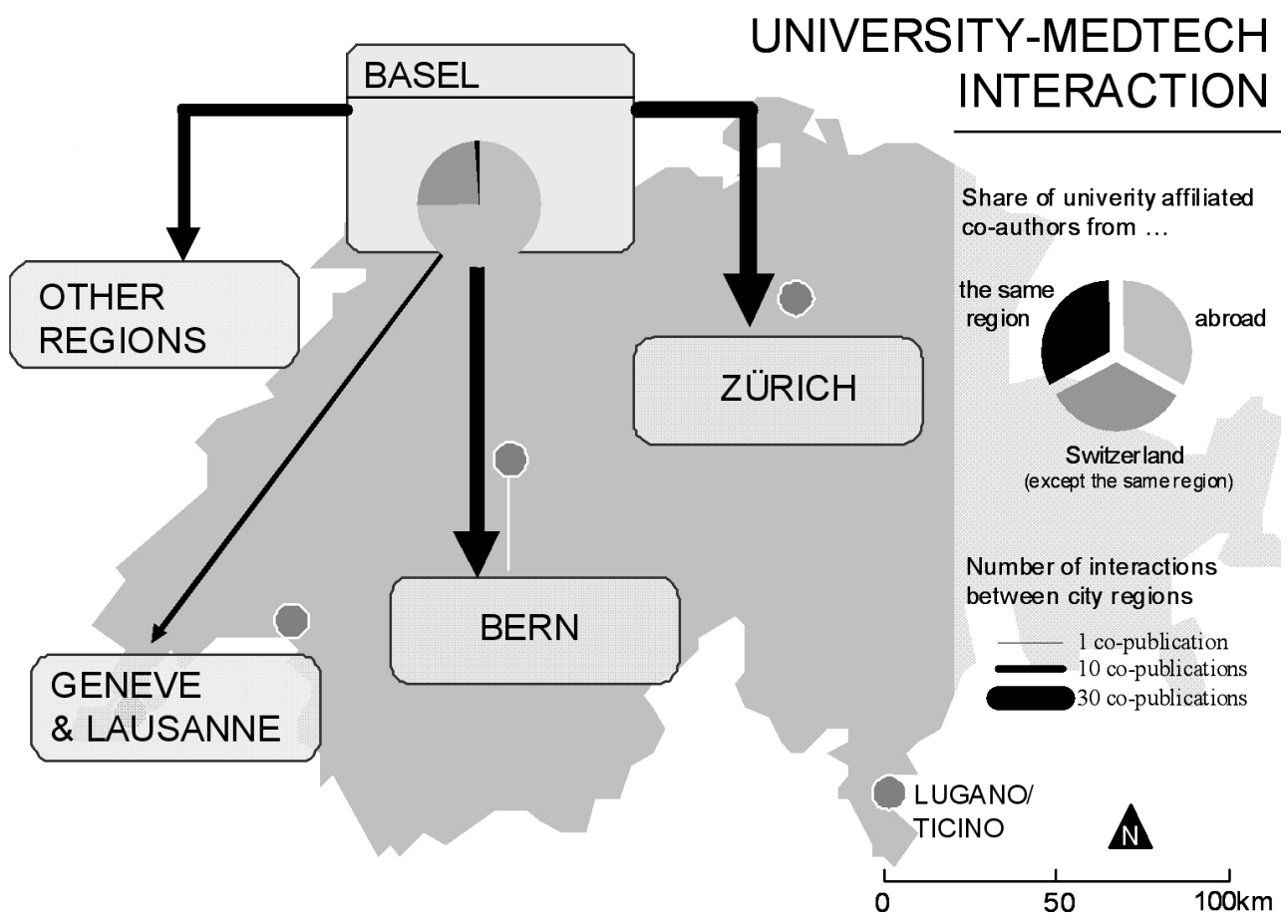

Source : calculs de l'auteur à partir de Web of Science 2006.

Les entreprises medtech de la région bâloise présentent moins de co-publications réalisées avec des partenaires privés qu'avec les universités. Cela est dû à la faible motivation de publier qui est à l'œuvre durant la phase d'ingénierie du processus d'innovation où les entreprises medtech interagissent fortement avec d'autres firmes (ce biais méthodologique est décrit au chapitre 3). Douze des 21 partenaires des copublications analysées sont localisés à l'étranger. Tous les autres partenaires (9) sont situées dans la région de Bâle, ce qui indique un avantage lié à la proximité spatiale en matière de transfert de connaissance non codifiables. La plupart des partenaires établis 
à l'étranger sont des filiales ou des émanations de la même entreprise, comme Synthes USA ou Cochlear SA USA.

\section{Conclusion}

57 Trois types d'interactions entre l'industrie pharmaceutique, d'une part, et les industries biotech et medtech, d'autre part, ont été observées.

58 1. Le premier type peut être considéré comme une influence indirecte et à long terme exercée sur le Système d'innovation suisse par l'industrie pharmaceutique, particulièrement par le biais des institutions qui font la promotion de l'excellence dans la recherche en sciences de la vie. Cet effet est manifeste sur l'ensemble de la Suisse, avec cependant plus d'intensité sur les villes qui possèdent de grands instituts et de grandes universités. Les résultats concrets de cette influence sont révélés par le nombre élevé d'entreprises biotech issues des universités à Lausanne et à Zurich et leurs interactions étroites avec les universités de leur région. Dans le cas des medtech, aucune preuve de l'influence indirecte de l'industrie pharmaceutique sur l'évolution de l'industrie medtech à Bâle ne peut être observée à long terme.

59 2. Une influence plus directe peut être observée dans le secteur biotechnologique par le biais des créations d'entreprises dans la région de Bâle. Cependant, la création de ces entreprises a largement été limitée à la période de restructuration de l'industrie pharmaceutique, ce qui signifie que cet effet pourrait ne pas se répéter. La présence de ces entreprises créées durant cette période particulière explique le classement de Bâle dans la catégorie des hotspots biotechnologiques européens par Ernst \& Young. A nouveau, aucune influence de ce type ne peut être observée pour les entreprises medtech mais des recherches plus approfondies sur d'autres localisations en Suisse devraient être réalisées.

60 3. Une troisième influence, indirecte et limitée au contexte régional, peut être observée. Contrairement aux deux précédentes, cette influence limite le développement des activités biotech et medtech: l'Université de Bâle n'a donné naissance qu'à un nombre limité d'entreprises et ne constitue que rarement un partenaire pour les entreprises biotech et presque jamais pour les entreprises medtech. Il se pourrait à ce sujet que les structures de recherche soient occupées par les entreprises pharmaceutiques, qui freinent les interactions possibles entre l'université et les firmes biotech ou medtech et rendent la création d'entreprises par les scientifiques peu probables.

61 Ces arguments montrent que l'influence de l'industrie pharmaceutique affecte les régions suisses de manière inégale. A ce titre, Bâle semble s'être développée d'une manière très différente des autres régions du pays. Les différentes structures entrepreneuriales conjuguées aux différentes interactions observées, notamment les contacts au sein du milieu des biotechnologies, pourraient laisser penser qu'il existe un Système d'innovation régional (RIS) spécifiquement bâlois. Les résultats présentés dans cet article suggèrent que cela n'est pas le cas, tout d'abord parce que les différences observées en termes de structure des entreprises biotech résultent de la restructuration de l'industrie pharmaceutique bâloise. Cet événement conjoncturel laisse penser que les différences régionales pourraient s'estomper à l'avenir. Ensuite, la faible importance des institutions formelles au niveau régional rend l'existence d'un 
RIS improbable. Bien que le canton de Bâle possède les compétences nécessaires à la mise en œuvre de politiques spécifiques, la région de Bâle demeure fortement fragmentée en termes administratifs. L'existence de trois pays (Suisse, France et Allemagne) et de quatre cantons contribue à ce que les institutions formelles n'aient pas développé de politique commune. Cependant, des recherches approfondies sur le rôle et l'extension géographique des autres facteurs principaux relatifs aux secteurs analysés ici, notamment les interactions informelles, permettraient de mieux connaître l'étendue du système d'innovation. En outre, une analyse des interactions de l'ensemble des entreprises medtech de Suisse apporterait une meilleure compréhension des spécificités du milieu medtech bâlois.

\section{BIBLIOGRAPHIE}

Arvanitis, S., Arx, J.V., Hollenstein, H., Sydow, N. (2004). - Innovationstätigkeiten in der Schweizer Wirtschaft. Eine Analyse der Ergebnisse der Innovationserhebung 2002. Secrétariat d'Etat à l'économie SECO, Rapport de conjoncture 24, Berne.

Asheim, B., Gertler, M. (2005). - « The geography of innovation: Regional innovation systems », in: Fagerberg, D., Mowery, D.C., Nelson, R.R. (dir.), The Oxford handbook of innovation, Oxford.

Asheim, B., Isaksen, A. (1997). - « Location, Agglomeration and Innovation: Towards Regional Innovation Systems in Norway? », European Planning Studies, n5(3): 299-330.

Basler Zeitung (2005). - Metrobasel Report, Supplément 8, Bâle.

Bathelt, H., Depner, H. (2003). - « Innovation, Institution und Region: Zur Diskussion über nationale und regionale Innovationssysteme »,Erdkunde, n57, p.126-143.

Bergier, J.-F. (1974). - Naissance et croissance de la Suisse industrielle, Berne.

Bergier, J.-F. (1984). - Histoire économique de la Suisse, Lausanne.

OFS - Office fédéral de la statistique (2005). - www.ofs.admin.ch. Accédé le 08.01.2006.

Boschma, R.A., Lambooy, J.G. (1999). - « Evolutionary economics in economic geography »Journal of Evolutionary Economics, n 9 , p.411-439.

Bürgin, A. (1958). - Geschichte des Geigy Unternehmens von 1758-1939. Ein Beitrag zur Basler Unternehmer- und Wirtschaftsgeschichte, Bâle.

Busset, T. (1997). - Chemie in der Schweiz: Geschichte der Forschung und der Industrie, Bâle.

Clayton-Matthews, A. (2001). - The medical device industry in Massachusetts, University of Massachusetts, Donahue Institute.

Coenen, L., Moodysson, J., Ryan, C., Asheim, B.T., Phillips, P. (2005) - Knowledge Bases and Spatial Patterns of Collaboration: Comparing the Pharma and Agro-food Bioregions Scania and Saskatoon. Papier présenté à la conférence « Bringing Science to Life », Université de Toronto, Canada, 29 avril 1er mai. 
Cooke, P. (1992). - « Regional Innovation Systems: Competitive Regulation in the New Europe $»$, Geoforum, n²3, p.365-382.

Cooke, P. (1998). - «Introduction: Origins of the Concept », in Braczyk, H., Cooke, P., Heidenreich, M. (dir), Regional Innovation Systems; Londres: UCL Press, p.2-25.

Cooke, P. (2002). - « Biotechnology clusters as regional, sectoral innovation systems »,International Regional Science Review, n²5, p.8-37.

Cooke, P. (2002). - Rational drug design and the rise of bioscience megacenters. Papier présenté à la Triple Helix 4 Conference, Copenhague, Danemark, 6 - 9 mai.

Cooke, P. (2003). - « Geographic clustering in the UK biotechnology sector », in Fuchs, G. (éd.), Biotechnology in comparative perspective, Londres et New York, p.76-97.

Cooke, P. (2003). - « The evolution of biotechnology in three continents: Schumpeterian or Penrosian? »,European Planning Studies, n¹1(7), p.757-764.

Cooke, P. (2005). - Global bioregions, capabilities and innovation system networks, Papier présenté à la Triple Helix 5 Conference « The capitalization of knowledge: cognitive, economic, social and cultural aspects », Turin, $18-21$ mai.

Cortright, J. et Mayer, H. (2002). - Signs of lie: The growth of biotechnology centers the U.S, Washington.

Credit Suisse (2004). - Wirtschaftsraum Basel. Struktur und Perspektiven. CS Economic \& Policy Consulting - Regional Analysis, Zurich.

Credit Suisse (2005). - Schweiz im Wandel - Branche als Bausteine des Wachstums. CS Economic Briefing 41 , Zurich.

Dosi, G., Nelson, R.R. (1994). - « An Introduction to Evolutionary Economics »Journal of Evolutionary Economics, ${ }^{\circ} 4$, p.153-172.

Dosi, G., Freeman, C., Nelson, R.R., Silverberg, G., Soete, L. (éds) (1988). - Technical change and economic theory, Londres.

Dosi, G. (1988). - « The nature of the innovation process », in Dosi, G., Freeman, C., Nelson, R.R., Silverberg, G., Soete L. (éds), Technical change and economic theory, Londres, p.221-238.

Dümmler, P. (2005). - Wissensbasierte Cluster in der Schweiz: Realität oder Fiktion? Das Beispiel der Medizinaltechnikbranche, Dissertation Nr. 16082, ETH Zürich.

Dümmler, P., Abegg, C., Kruse, C., Thierstein, A. (2004). - Standorte der innovativen Schweiz. Räumliche Veränderungsprozesse von High-Tech und Finanzdienstleistungen, Office fédéral de la statistique (OFS), Neuchâtel.

Edquist, C. (éd.) (1997). - Systems of innovation-Technologies, institutions and organisations, Londres et Washington.

Elias, J. (2000). - « Innovationsstandort Schweiz. Indikatoren- Innovationsprofil- internationaler Vergleich »,Die Volkswirtschaft, ${ }^{\circ} 3$, p.6-14.

Ernst \& Young (2003a). - Endurance. The European biotechnology report 2003. 10th Anniversary Edition, Cambridge.

Ernst \& Young (2003b). - Swiss biotech report, Bâle.

Ernst \& Young, SWX Swiss Exchange, Secrétariat d'Etat à l'économie (SECO), KTI, Osec, Institut für Geistiges Eigentum, Swiss Medtech (2005). - The Swiss Medtech Report. 
Etzkowitz, H., Leydesdorf, L. (2000), - « The dynamics of innovation: From national innovation systems and « mode 2 » to a triple helix of university-industry-government relations », Research Policy, n²9, p.109-123.

Fagerberg, D., Mowery, D.C., Nelson, R.R. (éds) (2005). - The Oxford handbook of innovation, Oxford.

Feldmann, M.P., Francis, J. (2003). - « Fortune favours the prepared region: The case of entrepreneuship and the capitol region biotechnology cluster ",European Planning Studies, n¹1(7), p.766-788.

Fonds national suisse FNS (2002). - 1952-2002. 50 ans du FNS, Berne.

Fuchs, G. (éd.) (2003). - Biotechnology in comparative perspective, Londres et New York.

Hicklin, M. (2005) - « Zwischen den Leuchtürmen wächst ein reiches Biotop »,Basler Zeitung, supplément 8, Bâle, p.24-27.

Hotz-Hart, B., Good, B., Küchler, C., Reuter-Hofer, A. (2003). - Innovation Schweiz. Herausforderungen für Wirtschaft und Politik, Zurich et Coire.

Kloepper, C. (2008). - Globale Netzwerke, Regionale Einflüsse: Die Struktur und Geographie von Innovationsprozessen in der Biotechnologie. Eine Analyse der Innovationsnetzwerke von Biotechnologieunternehmen in elf Clustern in der Schweiz, Deutschland, Frankreich und Grossbritannien. Non publié.

Kollreuter, C., Müller, U., Schoder, T., Merki, M. (2005). - « Life Sciences: Eine Erfolgsgeschichte »,Basler Zeitung, supplément 8, Bâle, p.6-11.

Lawton Smith, H. (2005). - « The Biotechnology industry in Oxfordshire: enterprise and innovation »,European Planning Studies, n²12(7), p.985-1002.

Lundvall, B-Å. (1992). - National systems of innovation: Towards a theory of innovation and interactive learning, Londres.

Malecki, E.J. (1990). - Technology and economic development. The dynamics of local, regional and national change, Harlow.

Malecki, E.J., Oinas, P. (éds) (1999). - Making connections. Technological learning and regional economic change, Aldershot.

Nelson, R.R., Winter, S.G. (1974). - « Neoclassical vs. evolutionary theories of economic growth: Critique and prospectus »,Economic Journal, n84, p.886-905.

Niosi, J., Bas, T.G. (2003). - « Biotechnology megacenters; Montreal and Toronto regional systems of innovation »,European Planning Studies, n¹1(7), p.789-804.

OECD (1999). - Modern Biotechnology and the OECD, OECD Observer, Policy Brief, disponible sous www.oecd.org/publications/pol_brief.

Plaut Economics (2005). - Bedeutung der Pharmaindustrie für die Schweiz, Etude pour le compte de Interpharma, Regensdorf, Bâle.

Polanyi, M. (1958). - Personal Knowledge: Towards a Post-Critical Philosophy, Londres.

Porter, M. (1990). - The competitive advantage of nations, New York.

Prevezer, M. (1997). - « The dynamics of industrial clustering in biotechnology »,Small Business Economics, n9, p.255-271.

Prevezer, M. (2000). - « Ingredients in the early development of U.S. biotechnology industry »,Small Business Economics, n¹7, p.17-29. 
Schätzl, L., Revilla-Diez, J. (éds). - Technological Change and Regional Development in Europe, Berlin.

The Economist (2006). - Desperate measures. America's health care crisis.

Université de Bâle (2006a). - UniNews. Roche fördert die Grundlagenforschung der Universität Basel. Disponible sous http://www.unibas.ch.

Université de Bâle (2006b). - UniNews. Einrichtung der Novartis-Professur für Molekulare und Systemische Toxikologie. Disponible sous http://www.unibas.ch.

Venture Valuation (2006). - Swiss Life Sciences Database. Accédé le 30.01.2006 sous www.swisslifesciences.ch.

Zeller (2001). - Globalisierungsstrategien - Der Weg von Novartis. Springer-Verlag, Berlin, Heidelberg, New York.

Zeller, C., Oßenbrügge, J. (2002). - « The Biotech Region Munich and the spatial organization of its networks ", in Schätzl, L. et Revilla-Diez, J. (éds), Technological Change and Regional Development in Europe, Berlin, p.233-249.

Zeller, C. (2004). - « North Atlantic innovative relations of Swiss pharmaceuticals and the importance of regional biotech arenas »,Economic Geography, n80(1), p.83-111.

\section{RÉSUMÉS}

Cet article traite de l'influence d'un secteur ou d'une industrie dans l'économie nationale et de la manière dont il/elle peut influencer des secteurs et des technologies connexes. L'exemple choisi est constitué par l'industrie pharmaceutique suisse. L'article suggère que cette industrie a façonné le développement et la distribution spatiale des secteurs qui lui étaient liés, tels que les biotechnologies (biotech) et les technologies médicales (medtech). Il est supposé que cette influence diffère significativement selon l'extension géographique. Elle est manifeste à l'échelon national, dans la mesure où les biotech et les medtech ont bénéficié d'institutions nationales façonnées par l'industrie pharmaceutique. Les effets de cette industrie devraient également se faire sentir au niveau régional, notamment à Bâle où l'industrie pharmaceutique est concentrée, par le biais des créations d'entreprises et des liens d'affaires avec l'industrie pharmaceutique. Ces aspects sont abordés dans le cadre théorique des systèmes d'innovation nationaux et régionaux, en termes d'évolution, de dépendance au sentier et d'interactions.

This paper is dealing with the influence of one dominant sector or industry within a national economy and how related sectors and technologies are influenced. Research objective is the pharmaceutical industry in Switzerland which we suggest shaped the development as well as the spatial distribution of related business sectors such as biotechnology and medical technology. We suppose that its influence differs significantly in terms of spatial reach, meaning that medtech and biotech have profiteered from national institutions shaped by the pharmaceutical industry. Furthermore, a regional impact should be proved in the Basel region where the pharmaceutical industry is concentrated through spin-offs and close business linkages with the pharmaceutical industry. Those suggestions are considered within the theoretical concepts of national and regional innovation systems in terms of evolution, path dependency and interactions.

Für den vorliegenden Beitrag wurden Innovationsnetzwerke von Biotechnologieunternehmen aus Strasbourg und Basel mittel bibliometrischer Verfahren gemessen, miteinander verglichen und in ihren regionalen Kontext gestellt. Die Ergebnisse zeigen, dass sich die Innovationsnetzwerke erheblich bezüglich ihrer Organisation und ihrer Geographie 
unterscheiden. Diese Unterschiede lassen sich unter anderem durch die Grösse der untersuchten Biotechnologiecluster, durch die Entstehung der Unternehmen und durch das institutionelle Umfeld erklären.

INDEX

Keywords : Bale, biotechnology, cluster, innovation, innovation systems, Strasbourg Schlüsselwörter : Basel, Biotechnologie, Cluster, Innovation, Innovationsnetzwerke, Strasbourg Mots-clés : Bâle, biotechnologie, cluster, innovation, réseaux innovants, Strasbourg

\section{AUTEURS \\ CHRISTOF KLÖPPER}

Amt für Wirtschaft und Arbeit Basel-Stadt - Utengasse 36 Postfach 4005 Basel christof.kloepper@bs.ch

TINA HAISCH

BAK Basel Economics - Gueterstrasse 82 CH-4053 Basel - tina.haisch@bakbasel.com 